\title{
Helicobacter pylori Infection in Children: What Does a Pediatrician Need to Know?
}

\author{
Srikanth P Kadyada
}

\begin{abstract}
Background: Helicobacter pylori infection is a ubiquitous gastric infection, seen in almost half of the world's population. Majority of them acquire the infection by the age of 10 years. However, only a small proportion become symptomatic.

Methods: This narrative review examines the clinical features, histology, diagnostic approach and treatment options in children as per recent updated guidelines and comparison vis-à-vis with adult's guidelines at appropriate places. In brief; peptic ulcer disease (PUD) is a main manifestation in children. While in adults, in addition to PUD, it is associated with MALToma and distal gastric adenocarcinoma. There is no specific symptom of $H$. pylori disease; the evaluation is a part of all the diagnostic studies of upper $\mathrm{Gl}$ symptoms. Confirmation requires histological presence of $\mathrm{H}$. pylori in the biopsy sample along with atleast one biopsy based test (rapid urease test and PCR). On confirmation; PUD, MALToma, iron refractory iron deficiency anemia, chronic ITP requires treatment based on antimicrobial susceptibility. Eradication is confirmed by a noninvasive test such as stool antigen or C-13 urea breath test (13C-UBT test). Persistence of infection in a symptomatic child requires second line antimicrobials.

Conclusion: H. pylori is becoming common infection in children with myriad presentations. Protocol based approach and planned treatment is necessary to eradicate and prevent complications.
\end{abstract}

Keywords: Anemia, Bleeding, Children, Gastritis, Helicobacter pylori, Peptic ulcer disease.

Pediatric Infectious Disease (2019): 10.5005/jp-journals-10081-1206

\section{INTRODUCTION}

Helicobacterpylori is a pathogen that is recently added to the growing list of microbes causing disease in humans. It fulfills the Koch's postulates as a cause of chronic active gastritis. In 1983, Marshal and Warren identified this curved bacterium in the stomach. Since then many trivial and fatal health conditions have been identified both in children and in adults. ${ }^{1}$ This organism is one of the few microbial agents associated with cancer; and according to World Health Organization, this is a class I oncogene. ${ }^{2}$ For this groundbreaking work, they were conferred with noble prize in medicine in the year 2005. This narrative review highlights the recent evidence of various associations of $H$. pylori in children and management of most common $\mathrm{H}$. pylori disease.

\section{Microbiology}

Helicobacter pylori is a spiral, microaerophilic, gram-negative, slow growing, flagellated bacterium. The surface of the bacterium is coated with urease and thermal shock proteins. The other virulence factors are vacuolating cytotoxin (VacA) and cytotoxin-associated antigen (CagA). These factors cause disease by helping bacteria thrive in hostile acidic environment, helping in motility, altering the osmotic factor in the host cells, and perpetuating local immunity, leading to chronic inflammatory and tissue damage.

Pathogenesis can be best understood in various stages through which it is executed in the human stomach mucosa. First step is the survival and entry of bacteria through the hostile acidic mucosa. This is overcome by urease in the $\mathrm{H}$. pylori, which converts urea into ammonium leading to neutral, favorable environment for bacteria to thrive. The second step is wading through the thick mucus, which is facilitated by 4-7 polar sheathed flagella, and arrival of bacterium in the vicinity of gastric epithelial cells. The third step involves attachment to gastric epithelial cellular surface with the help of various adhesins. They include neutrophil-activating protein $A$, heat shock protein 60 , blood group
Division of Pediatric Gastroenterology, Department of Pediatrics, Manipal Hospitals, Bengaluru, Karnataka, India

Corresponding Author: Srikanth P Kadyada, Division of Pediatric Gastroenterology, Department of Pediatrics, Manipal Hospitals, Bengaluru, Karnataka, India, Phone: +91 8284959288, e-mail: kpkantha@gmail.com

How to cite this article: Kadyada SP. Helicobacter pylori Infection in Children: What Does a Pediatrician Need to Know? Pediatr Inf Dis 2019; 1(2):58-61.

Source of support: Nil

Conflict of interest: None

antigen-binding adhesin, and sialic acid-binding adhesin. The fourth and final step is the infliction of damage to host cells and induction of chronic inflammatory response by the VacA and CagA, which in turn leads to all the clinical manifestation seen in this infection. Various proteins are involved in all the steps highlighted above, which in turn are regulated by genes of the $H$. pylori. Polymorphisms in the genes lead to varied clinical manifestations.

\section{Epidemiology and Risk Factors}

Half of the world's population is estimated to be infected with $H$. pylori. ${ }^{3}$ Of this, majority of the infected individuals live in low- and middle-income countries. The transmission is mainly oral-oral (kissing and intake of premasticated food) or feco-oral route and is widespread in poor socioeconomic status. Humans are the principle reservoirs of this bacterium. According to one report in low- and middle-income countries, nearly $65 \%$ of adolescents are infected with $\mathrm{H}$. pylori. ${ }^{4}$ The prevalence is low (1.2-12.2\%) in high-income countries (HICs), but a very high prevalence $(60-80 \%)$ has been reported in low- and middle-income countries., 5eroprevalence studies in India have shown that by 10 years of age more than $50 \%$ and by 20 years more than $80 \%$ of the population are infected., 


\section{Clinical Presentation}

Helicobacter pylori leads to a host of clinical conditions, of which some are definitive, while many are still under consideration, which requires further studies to confirm or refute. Among the definitive associations, peptic ulcer disease (PUP) which includes erosion of the stomach, duodenal ulcers, and gastric ulcers followed by MALT lymphoma and distal stomach adenocarcinoma are the important ones. Histologically confirmed active chronic gastritis in the presence of H. pylori is one of the most common finding in children. Additionally, growth failure, IRIDA, CITP, gastroesophageal reflux disease (GERD), etc. claimed have an association in the pediatric age-group. But the evidence is not definitive as studies favor either of the sides.

As highlighted in the earlier part of the review, a large portion of the population is infected with $\mathrm{H}$. pylori; interestingly majority of them are asymptomatic. The symptomatic children present with plethora of upper Gl symptoms. These include upper abdominal pain, upper Gl bleed due to duodenal and gastric ulcer, GERD, symptoms of dyspepsia, etc. These manifestations are not specific for $H$. pylori disease as many other upper GI disorders have similar presentation. To note, functional dyspepsia, food allergy and eosinophil GI disorders, celiac disease, and even upper GI Crohn's can have similar complaints. Thus, a given child would be evaluated for most likely cause of upper Gl symptoms rather than $H$. pylori alone, and investigations are directed toward all these disorders and $H$. pylori would be one among them.

\section{IRIDA Due to H. pylori Infection}

It has been postulated that $H$. pylori causes IDA, specifically in adolescent girls. The foremost reason among them is the presence of hemorrhagic lesions in the stomach or duodenum, leading to blood loss anemia. However, many studies have refuted this mechanism. ${ }^{9}$ Other potential mechanism is increased gastric $\mathrm{pH}$ due to chronic gastritis, which reduces Fe absorption. Reduction of ferric to ferrous form facilitates membrane transport. Due to higher $\mathrm{pH}$, this mechanism is hampered. Another hypothesized mechanism is that $H$. pylori might lead to IDA by sequestering and utilizing iron, thus competing with the human host. But there is no direct correlation between $H$. pylori infection and IDA as many recent studies have failed to demonstrate this. ${ }^{10}$ Thus, the recent guidelines suggest to evaluate (with invasive tests) for H. pylori gastritis only if anemia is refractory to iron supplementation.

\section{Chronic Idiopathic Thrombocytopenia}

Idiopathic thrombocytopenia (ITP) is an autoimmune disorder caused by antibody-mediated destruction of platelets and $H$. pylori is blamed as an inciting agent due to antigen mimicry between cag $A$ and platelet glycoprotein antigens. Some studies have suggested improvement with anti-Hp therapy. Based on these evidences, the recent European Society of Pediatric Gastroenterology, Hepatology and Nutrition (ESPGHAN) and North American Society of Pediatric Gastroenterology, Hepatology and Nutrition (NASPGHAN) guidelines recommend to apply noninvasive diagnostic testing for $H$. pylori. If positive, then perform invasive testing to confirm and treat accordingly.

\section{Recurrent Abdominal Pain and H. pylori}

Recurrent abdominal pain (RAP) is a global pediatric problem, and many theories have been put forth to explain its possible reasons. Among which $H$. pylori is considered to be one but the results are otherwise. Many studies have failed to confirm the association of RAP and H. pylori. Two major studies in Europe failed to show any positive association. ${ }^{11,12}$ Even the studies on eradication therapy for H. pylori did not support the causality of RAP. ${ }^{13,14}$ Similarly, a recent meta-analysis has failed to demonstrate any association. ${ }^{1}$ Based on these, the recommendation is not to test for $\mathrm{H}$. pylori in cases of clinically diagnosed functional abdominal syndrome in children.

\section{Diagnosis}

\section{When to Consider H. pylori Testing? (Whom to Test)}

The primary goal in evaluating a child is to identify the cause of Gl symptoms. Clinical manifestations of $H$. pylori disease overlap with many other upper $\mathrm{Gl}$ diseases. The available test can be classified into noninvasive (no endoscopy required) and invasive (endoscopybased test; Table 1). Noninvasive tests should not be applied for initial diagnosis and the evidence is against test and treat strategy (applying noninvasive tests and treating if results are positive). Therefore, endoscopy is a necessity, with demonstration of at least two biopsy-based positive tests, with histological evidence of chronic active gastritis with $H$. pylori positivity. ${ }^{15}$

\section{Microbiological Methods (How to Test)}

Multiple options are available for confirming the presence of H. pylori disease. ${ }^{16,17}$ However, before undertaking any test for $H$. pylori, parents and guardians should be asked about the drug intake during the last 4 weeks. Acid-suppressive therapy should be stopped 2 weeks and antibiotics 4 weeks prior to testing. If acidsuppressive therapy [specifically proton pump inhibitors (PPIs)] cannot be stopped due to the risk of recurrence of symptoms, to improve the sensitivity of the diagnostic test, changing to an $\mathrm{H}_{2}$-receptor antagonist with discontinuation of the drug at least 2 days before testing is suggested.

The gold standard in the diagnosis of $H$. pylori-associated disease demonstrated the presence of the organism in the culture of gastric biopsy sample. However, culture may not always be possible for routine clinical use, demonstration of $H$. pylori gastritis on histopathology with at least one other positive biopsy-based test such as the rapid urease test (RUT) or molecular assay (polymerase chain reaction (PCR) or fluorescence in situ hybridization (FISH)) is optimum for clinical case identification. ${ }^{15}$ According to the updated Sydney system, at least six gastric biopsies should be taken for the

Table 1: Tests for investigating Helicobacter pylori infection

\begin{tabular}{|c|c|}
\hline \multirow[t]{5}{*}{ I } & Invasive tests requiring endoscopy \\
\hline & $\begin{array}{l}\text { Biopsies and histology (sensitivity: 60-100; specificity: } \\
98-100 \text { ) }\end{array}$ \\
\hline & Rapid urease test (sensitivity: $85-95$; specificity: 95-100) \\
\hline & Bacterial culture (sensitivity: 90-95; specificity: 100) \\
\hline & PCR for bacterial DNA (sensitivity: 95; specificity: 95) \\
\hline \multirow[t]{6}{*}{ II } & Noninvasive tests \\
\hline & $\begin{array}{l}\text { Serum and whole blood antibody (sensitivity: } 56-100 \text {; } \\
\text { specificity: } 60-98 \text { ) }\end{array}$ \\
\hline & Saliva antibody \\
\hline & Urine antibody \\
\hline & Stool antigen (sensitivity: 88-95; specificity: 95-98) \\
\hline & $\begin{array}{l}{ }^{13} \mathrm{C} \text {-urea breath testing (sensitivity: } 88-95 \text {; specificity: } \\
95-100 \text { ) }\end{array}$ \\
\hline
\end{tabular}

$\mathrm{PCR}$, polymerase chain reaction

Source: Adapted from ESPGHAN and NASPGHAN 2016 guidelines 
initial diagnosis of $H$. pylori. ${ }^{18}$ Two biopsies each should be taken from the antrum $(2-3 \mathrm{~cm}$ from pylorus along less and greater curvature) and corpus ( $8 \mathrm{~cm}$ from cardia along less and greater curvature), one biopsy from incisura angularis, and one additional biopsy for RUT or molecular-based biopsy.

There is a strong recommendation for performing antimicrobial susceptibility of the infecting H. pylori strain(s) and the eradication treatment needed to be designed accordingly. Susceptibility testing should be with regard to the culture biopsy-based techniques (real-time PCR or FISH). Molecular methods, especially commercial available kits based on real-time PCR, are suitable to detect $H$. pylori and clarithromycin resistance in gastric biopsy specimens or gastric juice. Noninvasive tests should be used only to confirm the eradication of the organism. The test of choice is ${ }^{13} \mathrm{C}-U B \mathrm{BT}$, and monoclonal $H$. pylori antigen test is an alternative. There is no role for antibody-based tests for H. pylori in serum, whole blood, urine, and saliva, as this may not clarify whether the infection is a present one or was in the past.

In case of IRIDA, endoscopy needs to be done, as there are many other $\mathrm{Gl}$ causes such as celiac disease, inflammatory bowel disease, etc., which also needs to be simultaneously evaluated. However, in case of CITP, a noninvasive test (stool antigen testing or ${ }^{13} \mathrm{C}-\mathrm{UBT}$ ) is sufficient to screen and if the result is positive, then endoscopy needs to be performed to further confirm the infection. There is no evidence to suggest for investigations or treatment of $H$. pylori in children with short stature.

\section{TREATMENT}

\section{Who Needs Treatment?}

Per the recent ESPGHAN and NASPGHAN guidelines, treatment is recommended when $H$. pylori-positive PUD (duodenal ulcer, gastric ulcers, erosion of the gastric mucosa) is diagnosed. ${ }^{15}$ Along with this, when endoscopy is normal (no visualized lesion in the mucosa) and $H$. pylori tests are positive, anti-H. pylori treatment should be suggested. This should be done with complete education of the parents regarding the possibility of nonresolution of the $\mathrm{Gl}$ symptoms and potential side effects during treatment as well as drug resistance. The only benefit of this is that it would help in longterm prevention of complications related to H. pylori; however, this is not strongly backed by any evidence. Treating children infected with $\mathrm{H}$. pylori, whose first-degree relative has a history of gastric cancer, is no longer recommended. Treatment success is negatively affected if the strain is resistant to one of the antibiotics used. Thus, therapies should be based on knowledge of antibiotic susceptibility testing. Most of the regimens are for 14 days. Proton pump inhibitor monotherapy may be continued after eradication therapy for another 2-4 weeks in patients with PUD. Various medications that can be used for treating H. pylori infection, along with dose, duration, and the alternative options, are given in Tables 2 and 3. The other potential treatment indications are IRIDA and cITP (all these need invasive tests to confirm the diagnosis). The algorithm in approaching a child with suspected $H$. pylori disease is shown in Flowchart 1.

Successful H. pylori eradication is associated with the cure of PUD and very low risk of relapse. Therefore, monitoring the success of the therapy is mandatory in these patients after 4-6 weeks of stopping antibiotics and at least 2 weeks after stopping PPI therapy. In case of failure to eradicate, child should receive rescue therapy. Success of treatment should be tested with noninvasive tests.
Table 2: Standard dosing regimen

\begin{tabular}{llll}
\hline Drug & Body weight & $\begin{array}{l}\text { Morning } \\
\text { dose }(\mathrm{mg})\end{array}$ & $\begin{array}{l}\text { Evening } \\
\text { dose }(\mathrm{mg})\end{array}$ \\
\hline PPI & $15-24$ & 20 & 20 \\
& $25-34$ & 30 & 30 \\
Amoxicillin & $>35$ & 40 & 40 \\
& $15-24$ & $500(750)^{*}$ & $500(750)^{*}$ \\
Clarithromycin & $25-34$ & $750(1,000)^{*}$ & $750(1,000)^{*}$ \\
& $>35$ & $1,000(1,500)^{*}$ & $1,000(1,500)^{*}$ \\
Metronidazole & $15-24$ & 250 & 250 \\
& $>35-34$ & 500 & 250 \\
& $25-34$ & 500 & 500 \\
Bismuth & $>35$ & 250 & 250 \\
& $>10$ & 500 & 250 \\
& $>10$ & 500 & 500 \\
\hline
\end{tabular}

*High-dose amoxicillin regimen

Source: Adapted from ESPGHAN and NASPGHAN 2016 guidelines

Table 3: Recommended options for first-line therapy for Helicobacter pylori infection

\begin{tabular}{ll}
\hline $\begin{array}{l}\text { Helicobacter pylori } \\
\text { antimicrobial susceptibility }\end{array}$ & Suggested treatment \\
\hline $\begin{array}{l}\text { Known } \\
\text { Susceptible to CLA } \\
\text { and to MET }\end{array}$ & $\begin{array}{l}\text { PPI-AMO-CLA 14 days with } \\
\text { standard dose }\end{array}$ \\
Resistant to CLA, & PPI-AMO-MET 14 days or \\
susceptible to MET & bismuth based \\
Resistant to MET, & PPI-AMO-CLA 14 days or \\
susceptible to CLA & bismuth based \\
Resistant to CLA & PPI-AMO-MET 14 days with high \\
and MET & dose for amoxicillin (Table 2) or \\
& bismuth based \\
Unknown & High-dose PPI-AMO-MET 14 days \\
& or bismuth based \\
\hline
\end{tabular}

CLA, clarithromycin; MET, metronidazole; AMO, amoxicillin; PPI, proton pump inhibitor

Source: Adapted from ESPGHAN and NASPGHAN 2016 guidelines

The test of choice is ${ }^{13} \mathrm{C}$-UBT and also the performance of stool antigen test is equally good. In centers where UBT is not available, stool antigen test can be performed.

\section{Conclusion}

This review highlights the recent evidence-based recommendations on various aspects of $H$. pylori management in children. Evaluation for upper Gl symptoms is the first step in diagnosis. Endoscopy is a standard testing procedure in the diagnosis of $H$. pylori disease in children. Both IRIDA and CITP are the two extra intestinal indications for treatment of $H$. pylori infection. When antimicrobial susceptibility testing is available, one needs to perform susceptibility test before instituting medications. All treated patients should undergo noninvasive test (UBT or stool antigen) to confirm eradication. 
Flowchart 1: How to arrive at a diagnosis of Helicobacter pylori infection in children and adolescents. Various tests for possible cause of gastrointestinal $(\mathrm{Gl})$ disorders

1. Upper abdominal pain

2. Upper GI bleed (ulcer bleed)

4. Iron refractory/iron deficiency anemia

3. cITP

4. Retrosternal burning and vomiting

Tests for possible causes of various $\mathrm{Gl}$ disorders

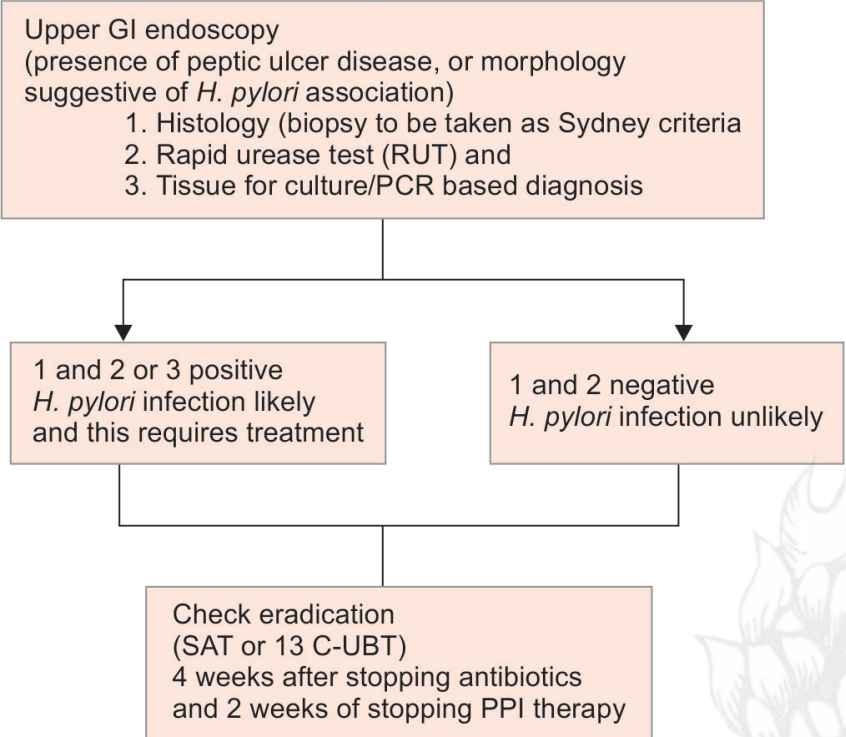

\section{HighLights}

- Nearly half of the world's population is infected with H. pylori.

- Only minority of them develop symptoms.

- In children, PUD is the most common manifestation. Both IRIDA and CITP are the extraintestinal manifestations requiring evaluation and treatment of $H$. pylori infection.

- There is no association between growth failure and RAP in children.

- Diagnosis should be based on invasive tests, and all treated patients should UBTs to confirm eradication.

- Antimicrobial susceptibility testing should be done when available to select appropriate medications.

\section{References}

1. Spee LAA, Madderom MB, Pijpers M, et al. Association between helicobacter pylori and gastrointestinal symptoms in children. Pediatrics 2010;125(3):e651-e669. DOI: 10.1542/peds.2010-0941.

2. Amieva M, Peek RM. Pathobiology of Helicobacter pylori-induced gastric cancer. Gastroenterology 2016;150(1):64-78. DOI: 10.1053/ j.gastro.2015.09.004.
3. Suerbaum S, Michetti P. Helicobacter pylori infection. N Engl J Med 2002;347(15):1175-1186. DOI: 10.1056/NEJMra020542.

4. Kato S, Sherman PM. What is new related to Helicobacter pylori infection in children and teenagers? Arch Pediatr Adolesc Med 2005;159(5):415-421. DOI: 10.1001/archpedi.159.5.415.

5. Wangda S, Richter JM, Kuenzang P, et al. Epidemiology of Helicobacter pylori infection in asymptomatic schoolchildren in Bhutan. Helicobacter 2017;22(6). DOI: 10.1111/hel.12439.

6. Ahmad MM, Ahmed DS, Rowshon AHM, et al. Long-term re-infection rate after Helicobacter pylori eradication in Bangladeshi adults. Digestion 2007;75(4):173-176. DOI: 10.1159/000107046.

7. Graham DY, Adam E, Reddy GT, et al. Seroepidemiology of Helicobacter pylori infection in India. Comparison of developing and developed countries. Dig Dis Sci 1991;36(8):1084-1088. DOI: 10.1007/ BF01297451.

8. Gill HH, Majmudar P, Shankaran K, et al. Age-related prevalence of Helicobacter pylori antibodies in Indian subjects. Indian J Gastroenterol 1994;13(3):92-94.

9. DuBois S, Kearney DJ. Iron-deficiency anemia and Helicobacter pylori infection: a review of the evidence. Am J Gastroenterol 2005;100(2):453-459. DOI: 10.1111/j.1572-0241.2005.30252.x.

10. Sarker SA, Mahmud H, Davidsson L, et al. Causal relationship of Helicobacter pylori with iron-deficiency anemia or failure of iron supplementation in children. Gastroenterology 2008;135(5): 1534-1542. DOI: 10.1053/j.gastro.2008.07.030.

11. Bode $\mathrm{G}$, Rothenbacher D, Brenner $\mathrm{H}$, et al. Helicobacter pylori and abdominal symptoms: a population-based study among preschool children in southern Germany. Pediatrics 1998;101(4 Pt 1):634-637. DOI: 10.1542/peds.101.4.634.

12. Tindberg $\mathrm{Y}$, Nyrén $\mathrm{O}$, Blennow $\mathrm{M}$, et al. Helicobacter pylori infection and abdominal symptoms among Swedish school children. J Pediatr Gastroenterol Nutr 2005;41(1):33-38. DOI: 10.1097/01. MPG.0000163734.84518.9E.

13. Oderda G, Dell'Olio D, Morra I, et al. Campylobacter pylori gastritis: long term results of treatment with amoxycillin. Arch Dis Child 1989;64(3):326-329. DOI: 10.1136/adc.64.3.326.

14. Ashorn M, Rägö T, Kokkonen J, et al. Symptomatic response to Helicobacter pylori eradication in children with recurrent abdominal pain: double blind randomized placebo-controlled trial. J Clin Gastroenterol 2004;38(8):646-650. DOI: 10.1097/01. mcg.0000135366.58536.b3.

15. Jones NL, Koletzko S, Goodman K, et al. Joint ESPGHAN/NASPGHAN Guidelines for the management of Helicobacter pylori in children and adolescents (update 2016). J Pediatr Gastroenterol Nutr 2017;64(6):991-1003. DOI: 10.1097/MPG.0000000000001594.

16. Guarner J, Kalach N, Elitsur Y, et al. Helicobacter pylori diagnostic tests in children: review of the literature from 1999 to 2009. Eur J Pediatr 2010;169(1):15-25. DOI: 10.1007/s00431-009-1033-x.

17. Lopes Al, Vale FF, Oleastro M. Helicobacter pylori infection - recent developments in diagnosis. World J Gastroenterol 2014;20(28): 9299-9313. DOI: 10.3748/wjg.v20.i28.9299.

18. Dixon MF, Genta RM, Yardley JH, et al. Classification and grading of gastritis. The updated Sydney System. International Workshop on the Histopathology of Gastritis, Houston 1994. Am J Surg Pathol 1996;20(10):1161-1181. DOI: 10.1097/00000478-19961000000001. 